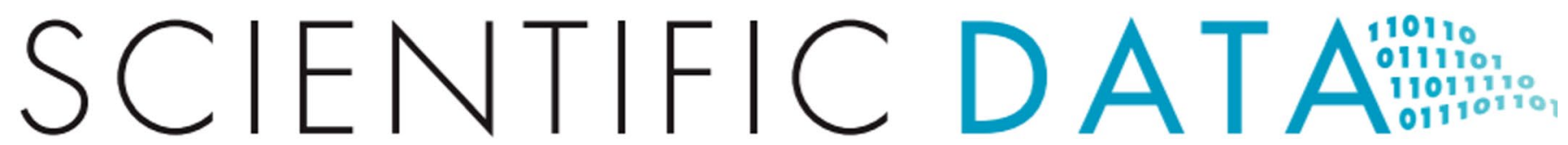

Check for updates

OPEN

DATA DESCRIPTOR

\title{
A global sea state dataset from spaceborne synthetic aperture radar wave mode data
}

\author{
Xiao-Ming Li $\mathbb{1}^{1 凶}$ \& BingQing Huang ${ }^{1,2}$ \\ This dataset consists of integral sea state parameters of significant wave height (SWH) and mean wave \\ period (zero-upcrossing mean wave period, MWP) data derived from the advanced synthetic aperture \\ radar (ASAR) onboard the ENVISAT satellite over its full life cycle (2002-2012) covering the global \\ ocean. Both parameters are calibrated and validated against buoy data. A cross-validation between the \\ ASAR SWH and radar altimeter (RA) data is also performed to ensure that the SAR-derived wave height \\ data are of the same quality as the RA data. These data are stored in the standard NetCDF format, \\ which are produced for each ASAR wave mode Level1B data provided by the European Space Agency. \\ This is the first time that a full sea state product in terms of both the SWH and MWP has been derived \\ from spaceborne SAR data over the global ocean for a decadal temporal scale.
}

\section{Background \& Summary}

The sea state is one of the key parameters of the "essential climate variables" (ECVs) defined by the Global Climate Observing System (GCOS) to meet the requirements of the climate change community. Spaceborne radar measurements of the sea state in terms of the significant wave height (SWH) and mean wave period (MWP), particularly from radar altimeters (RAs), have been available for a few decades ${ }^{1}$. Long-term RA measurements can reflect some wave height trends in the global oceans, and these trends might be associated with climate change ${ }^{2}$. Another radar sensor capable of measuring the sea state is known as spaceborne synthetic aperture radar (SAR), which became available at the same time as RAs; consequently, both instruments were on board the Seasat ${ }^{3}$ satellite launched in 1978. However, unlike nadir-looking RAs, SAR is a side-looking radar, which allows SAR to image large surface areas. Additionally, SAR can achieve a high spatial resolution in the azimuth (flight) direction through the "aperture synthetizing" technique ${ }^{4}$. In principle, spaceborne SAR should be able to effectively measure the sea state from space, as this technology images sea surface waves in two dimensions ${ }^{5}$, at a high spatial resolution. However, as surface waves are in motion during the SAR imaging time (i.e., water particles are moving either toward or away from the radar system), the high-frequency components of ocean waves are missed (the "cut-off" effect), and the distortion of the spectrum occurs during the imaging process of SAR ${ }^{6,7}$. Therefore, the SAR imaging of surface gravity waves is generally considered a nonlinear process ${ }^{8}$, complicating the retrieval of ocean wave parameters from SAR data. Two-dimensional wave spectra predicted by ocean wave modeling (e.g., $\mathrm{WAM}^{9}$ ) or derived from other sources ${ }^{10}$ must commonly be used as a priori information (also called the "first guess") in the retrieval ${ }^{11}$ to compensate for the lost and distorted ocean wave information during SAR imaging. However, as a result of this compensatory approach, the retrieval of ocean wave parameters from SAR data has to rely on the priori information, which significantly limits SAR as an independent remote sensing instrument that can measure the sea state.

The wave mode (WM), which is dedicated to measurements of ocean wave, is a unique imaging mode of SAR. Although the WM covers a relatively small area of the sea surface (approximately $6 \mathrm{~km}$ by $10 \mathrm{~km}$ ), these data are automatically acquired by spaceborne SAR over the global oceans. From the European Remote Sensing

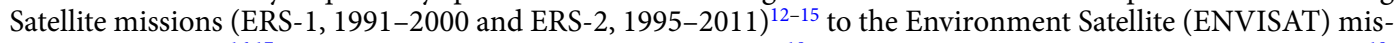
sion $(2002-2012)^{16,17}$ and the current Sentinel-1A/1B (2014 - $)^{18}$ and the Chinese Gaofen-3 (2016 -) missions ${ }^{19}$, WM data have been available for nearly 30 years and will continue to be acquired into the future, constituting a valuable dataset for global sea state measurements. On the basis of SAR WM data, some interesting investigations of global ocean waves, particularly with respect to the dynamics of ocean swells ${ }^{20-22}$, have been reported. Such 

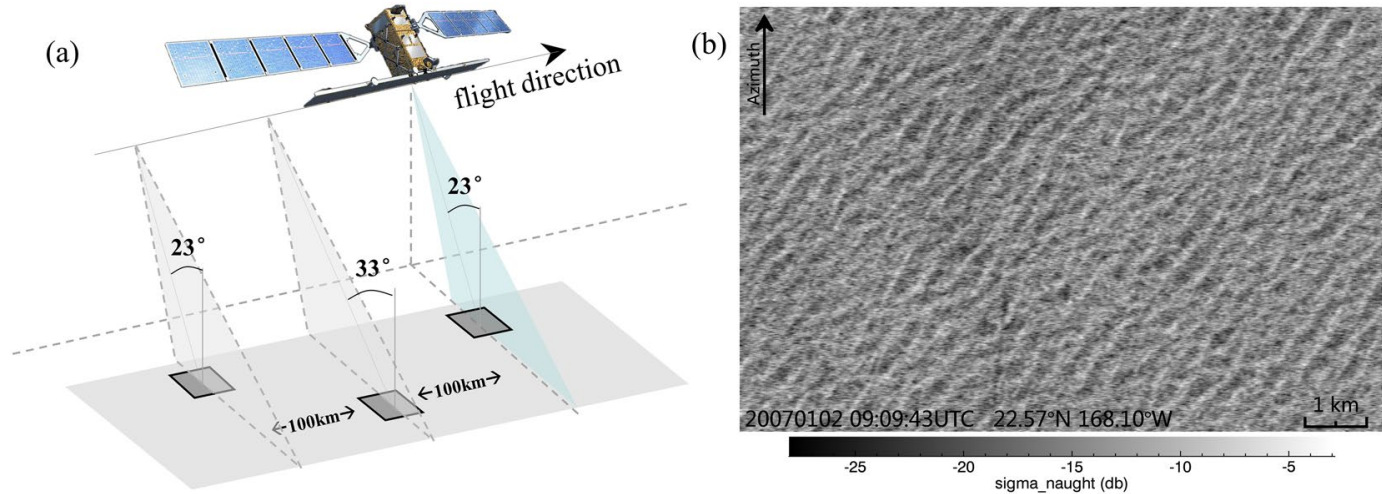

Fig. 1 (a) Geometry of the ASAR WM data acquisitions. Note that the WM data with an incidence angle of $33^{\circ}$ acquired by ASAR were only available during experiments (refer to the main text for details). (b) is an example of ASAR WM images acquired over the ocean. The acquisition date and location are marked on the image.

analyses can be performed because ocean swells are generally considered linearly or quasi-linearly imaged by SAR; thus, the abovementioned nonlinear inversion process can be degraded to a quasilinear approach ${ }^{23,24}$, in which case a priori information is no longer needed. However, such a quasilinear inversion cannot yield full sea state parameters of both windsea (wind waves) and swell, and instead yields the sea state parameters of swell, or more accurately called the parameters of the ocean wave components imaged by spaceborne SAR. Therefore, to overcome such a weakness, various parametric models that directly relate SAR-measured sea surface radar backscatter (radar cross section) to the full sea state parameters of SWH and MWP of both windsea and swell have been proposed $^{14,17,18}$, which also do not need a priori information and can provide independent SAR measurements of global ocean waves. Here, we developed a global sea state dataset from the WM data acquired by the advanced synthetic aperture radar (ASAR) onboard the satellite ENVISAT from 2002 to 2012 based on the parametric model "CWAVE_ENV"17. This is the first time that a global ocean dataset of full sea state parameters in terms of both SWH and MWP in a decadal temporal scale has become publicly available based on spaceborne SAR data and we believe that this dataset, in conjunction with RA datasets that are widely exploited at present, is valuable for global observations of ocean waves. On the other hand, full sea state parameters also become available in the Sentinel-1 WM data based on the similar retrieval method to the CWAVE type algorithms. Therefore, by combining both the historical ASAR WM ocean wave product and the current continuously obtained Sentinel-1 WM product, one can expect a long-term spaceborne SAR sea state dataset available for global ocean observations.

\section{Methods}

ASAR WM data. In the ERS-1 and ERS-2 missions, the SAR WM data were publicly available in the formation of two-dimensional image spectra in discrete formats, i.e. allocating the image spectrum energy in numbers of directional and frequency bins ${ }^{25}$. Beginning with the ENVISAT mission, the ASAR WM data in single look complex format ${ }^{26}$ (i.e., consisting of a real part $\mathrm{R}_{e}$ and an imaginary part $\mathrm{I}_{m}$ ) were provided to users; these data record both the magnitude and phase of the returned radar signals. The SAR image intensity $\left.{ }_{I}\right)$ is therefore calculated as $I=R_{e}^{2}+I_{m}^{2}$. By performing a radiometric calibration of intensity data, the normalized radar cross section, denoted $\sigma_{0}$, can be obtained and then used to retrieve sea state parameters.

ASAR WM data have a spatial coverage ranging from $6 \mathrm{~km} \times 5 \mathrm{~km}$ to $10 \mathrm{~km} \times 5 \mathrm{~km}$ over the sea surface. The distance between two consecutive acquisitions of WM data is $100 \mathrm{~km}$, which could be seen from the image geometry of ASAR WM in Fig. 1(a). One example of ASAR WM data acquired over the ocean is shown in Fig. 1(b), which clearly displays patterns of ocean waves (swells).

The parametric model "CWAVE_ENV" was applied to the ASAR WM data to generate the sea state parameters of SWH $\left(H_{s}\right)$ and MWP $\left(T_{m 02}\right)$. This type of parametric model was first proposed for the reprocessed ERS-2 WM data ${ }^{14}$; the name "CWAVE" indicates the use of a C-band (SAR) wave retrieval algorithm, like the widely used C-band geophysical model function "CMOD" 27 to retrieve sea surface wind fields from scatterometer and SAR data. Because the development and validation of parametric models have been described in detail in previous studies ${ }^{14,17}$, only the rationale for using the parametric model is discussed here.

Although imaging mechanisms of ocean surface gravity waves by SAR remain to be further investigated, the measured radar backscatter from the sea surface is closely related to various sea state parameters (denoted $W$ ) through relations with a set of parameters (expressed as a vector, $S\left(s_{1}, \ldots, s_{n s}\right)$ ). These parameters can be directly derived from SAR data, as expressed in Eq. (1).

$$
W=a_{0}+\sum_{1 \leq i \leq n_{s}} a_{i} s_{i}+\sum_{1 \leq i \leq j \leq n_{s}} a_{i, j} s_{i} s_{j}
$$

In the above model, the sea state parameter $W$ is expressed as a linear combination of a number of $n_{s}$ ASAR image parameters $\boldsymbol{S}\left(s_{1}, \ldots, s_{n s}\right)$ with the extended coefficient vector $\boldsymbol{A}\left(a_{0}, \ldots, a_{n s}, a_{11}, \ldots, a_{n_{s}} a_{n_{s}}\right)$. To also include nonlinearities as well as possible coupling among different parameters, a quadratic term is added to the equation (the third term in the equation). There are 22 ASAR image parameters (i.e., $n_{s}=22$ ) used in the CWAVE_ENV model. 
Two parameters of the normalized radar cross section $\sigma_{0}$ and the variance $c v s r$, are directly calculated from the intensity data. The remaining 20 parameters are derived from the FFT spectrum of the ASAR WM intensity image. The major reason for using the spectral parameters is that the traditional nonlinear or quasi-linear retrievals connect the SAR image spectrum with two-dimensional ocean wave (or swell) spectra. On the other hand, $\sigma_{0}$ is closely related with the wind speed (e.g., represented by the CMOD functions), and therefore, the information of windsea on SAR images is also involved in this equation. This is the general rationale that the function can represent both swell and windsea information.

The least-square minimization approach is used to determine the coefficient vector $A$ which consists of a number of $n_{A}$ coefficients, as defined in Eq. (2), where $\left(w^{(1)}, S^{(1)}\right), \ldots,\left(w^{(N)}, \ldots, S^{(N)}\right)$ represents the available data pairs of SAR image parameters and the collocated tuning dataset of the integral sea state parameter (e.g., SWH or MWP).

$$
J_{\text {cost }}(A)=\sum_{j=1}^{N}\left(w^{(j)}-\sum_{i=0}^{n_{A}-1} \boldsymbol{A}_{i} \boldsymbol{S}_{i}^{j}\right)^{2}
$$

After the coefficient vector $A$ is determined, one can derive the SWH or MWP directly from ASAR WM data using Eq. (1). The preliminary validation of the ASAR-derived $h_{s}$ values using the CWAVE_ENV algorithm was conducted for a two-month (January and February 2017) dataset. Comparisons with the National Data Buoy Center (NDBC) in situ buoy measurements yielded a bias of $0.06 \mathrm{~m}$ and a root-mean-squared-error (RMSE) of $0.70 \mathrm{~m}^{17}$. Here, we applied this parametric model to the entire dataset of the ASAR WM data of its full life cycle.

The entire ENVISAT mission ranged from March 2002 to April 2012. The ASAR data that we received from the European Space Agency (ESA) cover the period from December 2002 to April 2012. During the lifetime of ENVISAT, the ASAR instrument acquired WM data in vertical-vertical (VV) polarization with an incidence angle of $23^{\circ}$, except during two experimental periods, in which the acquired WM data had an incidence angle of approximately $33^{\circ}$. The first period ranged from January $24^{\text {th }}$ to February $6^{\text {th }}, 2007$, and the second one ranged from March $6^{\text {th }}$ to March $13^{\text {th }}, 2007$. From January $24^{\text {th }}$ to January $30^{\text {th }}, 2007$, the WM data were acquired in horizontal-horizontal $(\mathrm{HH})$ polarization. In addition to excluding the WM data acquired during these two experimental periods, the following criteria were applied to further screen the data.

(i) The ASAR WM data acquired in polar regions were excluded from further processing because they might be affected by sea ice; thus, only the data acquired between $65^{\circ} \mathrm{S}$ and $70^{\circ} \mathrm{N}$ were used to generate sea state parameters.

(ii) Although ASAR WM images have a relatively small spatial coverage compared with images acquired in other modes, e.g., the imaging mode and wide swath mode, the WM images are also affected by other sea surface features not related with ocean waves, e.g., oil spills, atmospheric features, and bright targets. To select only ASAR WM images that display a homogeneous sea surface (e.g., the case shown in Fig. 1b) and derive sea state parameters, some parameters were used for automatic detection. We previously used the "homogeneity factor" 28 to classify ASAR WM images into homogeneous and inhomogeneous classes; if the sea surface is purely homogeneous, this factor is equal to 1 . Through the visual inspection of large amounts of both ERS-2/SAR and ENVISAT/ASAR WM data, the homogeneity factor was set to 1.05 as a threshold for selecting appropriate ASAR WM data for retrieval. Approximately $94.42 \%$ of the data have a homogeneity factor lower than 1.05 , which are treated as good data for deriving sea state parameters. However, the trade-off is that for WM data with a homogeneity parameter higher than 1.05 may also present an acceptable situation of retrieval. Therefore, we lowered the threshold of the homogeneity factor to 1.50 in order to process more data to sea state parameters, but the data with a homogeneity factor in the range between 1.05 and 1.50 are flagged as "suspect" for further investigation, which is described in detail in Data Records. It should be noted that only the WM data with a homogeneity parameter less than 1.05 were used for calibration and validation presented in the following. After the aforementioned preprocessing steps, approximately 6.69 million ASAR WM data were used to generate global ocean wave parameters.

In situ buoy data. In situ buoy measurements of sea state parameters were used to validate and calibrate the retrieved SWH and MWP based on the ASAR WM data. The GlobWave project (http://globwave.ifremer.fr/) collected a large amount of in situ buoy data from several buoy networks. Among the different buoy datasets, it is found that the one provided by the European Center for Medium-Range Weather Forecasts (ECMWF) contains more data ( 649 buoys collected between 2002 and 2012) than any of the other datasets. It is a comprehensive collection of buoy data from various networks including NDBC, the Marine Environmental Data Section (MEDS), the Coastal Data Information Program (CDIP) and others. Therefore, we selected the ECMWF-provided buoy data (hereafter referred to as "ECMWF buoy data") for the evaluation and calibration of the ASAR-derived SWH.

The ASAR-retrieved MWP is the zero-upcrossing mean wave period $\left(T_{m 02}\right.$, also often denoted $\left.T_{z}\right)$, as defined in Eq. 3. One can find that both the definitions of $H_{s}$ and $T_{m 02}$ are in a consistent manner relating to zero-upcrossing waves. Both are the two widely used parameters to describe sea state. We found that many recorded MWP data in the ECMWF buoy dataset are with values of zero, in contrast, the corresponding NDBC spectral data are normal. Therefore, we used the NDBC two-dimensional buoy spectrum (also accessed from the GlobWave data portal, hereafter called "NDBC buoy data") to calculate $T_{m 02}$ for comparison with the ASAR-retrieved MWP. The quality flag of the NDBC buoy spectral data in the GlobWave data portal is named spectral_wave_density_qc_level. The values of this flag are $0,1,2,3$ and 4, which represent unknown, unprocessed, bad, suspect and good, respectively. We used only the good wave spectral data for calibration and validation. 


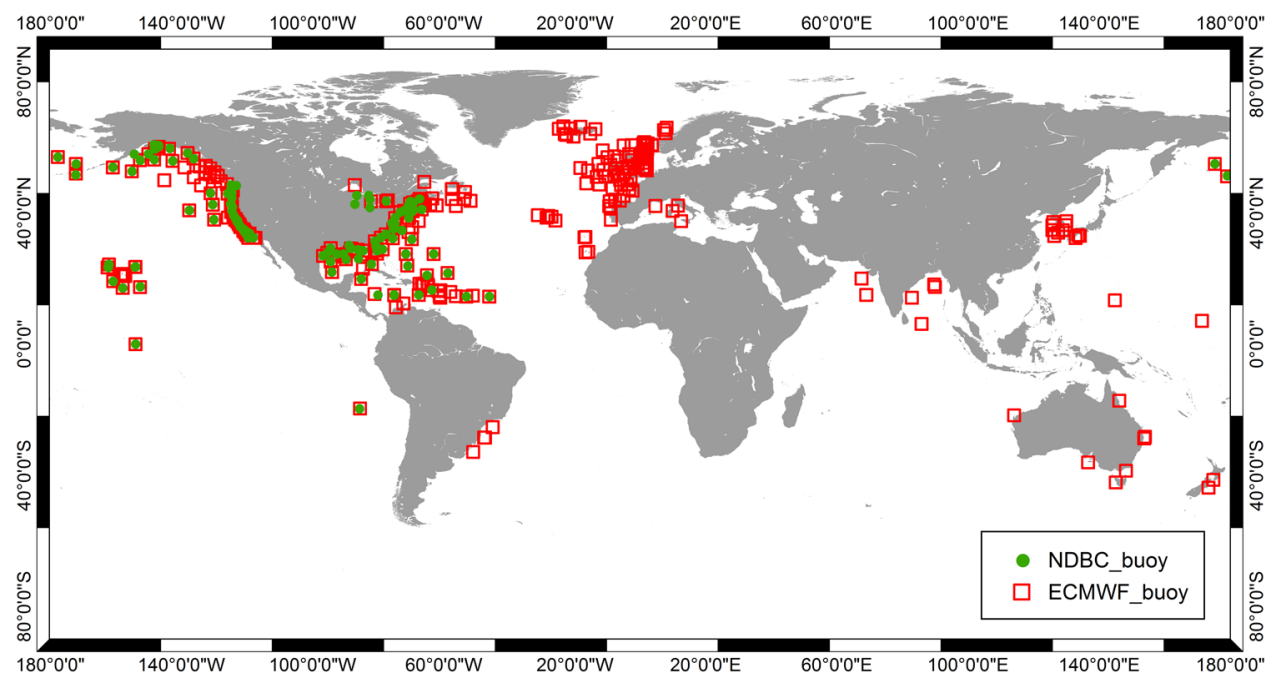

Fig. 2 Locations of the collocated ECMWF buoys (red squares) and NDBC buoys (green dots).
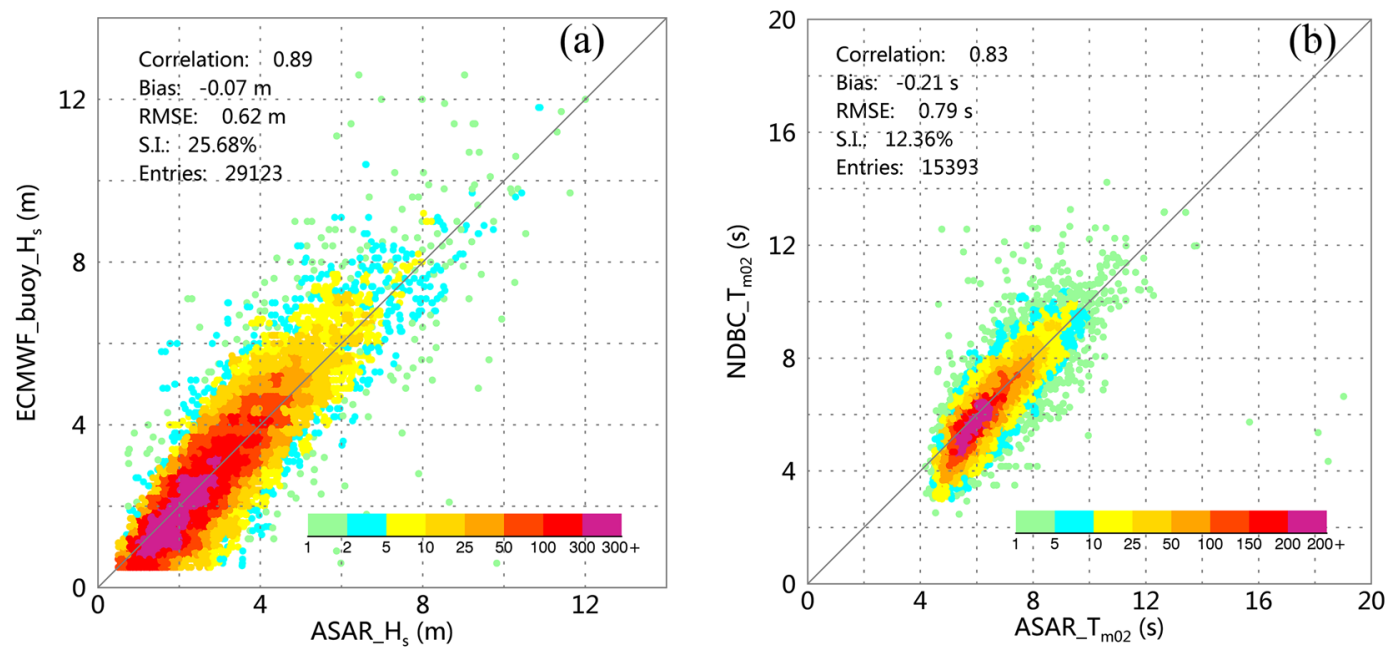

Fig. 3 (a) Comparison between the ASAR-derived SWH and the ECMWF buoy data. (b) Comparison between the ASAR-derived MWP and the NDBC buoy data.

$$
\begin{aligned}
& T_{m 02}=\sqrt{m_{0} / m_{2}} \\
& m_{n}=\sum_{i} f_{i}^{n} S_{i} \Delta f_{i}
\end{aligned}
$$

In the above equations, $\mathrm{m}_{n}$ is the $n^{\text {th }}$ spectral moment, $f_{i}$ is the $i^{\text {th }}$ discrete frequency, $\Delta f_{i}$ is the width of the $i^{\text {th }}$ discrete frequency and $S_{i}$ is the spectral density over the $i^{\text {th }}$ frequency.

Both the ECMWF and NDBC buoy data were collocated with the ASAR WM data following the criteria that the temporal difference is less than 30 minutes and the spatial distance is less than $100 \mathrm{~km}$. For cases in which several buoys satisfied the collocation criteria, only the measurements from the buoy nearest to the corresponding ASAR WM data location were used for the validation and calibration. Locations of the collocated ECMWF buoys and NDBC buoys data are shown in Fig. 2.

Comparison of ASAR retrievals with buoy wave data. To compare the ASAR-derived SWH (denoted ASAR_ $H_{s}$ ) with the ECMWF buoy SWH (denoted ECMWF_buoy_ $H_{s}$ ), we limited the SWH to the range from $0.5 \mathrm{~m}$ (to avoid the biased retrievals induced by the very low radar backscatter of the sea surface) to $30.0 \mathrm{~m}$. Eventually, 29,123 data pairs were retained for comparison, and the corresponding scatter diagram is shown in Fig. 3(a). With respect to the comparison of MWP, the NDBC buoy MWP (denoted NDBC_ $T_{m 02}$ ) are all larger than $2.0 \mathrm{~s}$ but smaller than $20.0 \mathrm{~s}$. Eventually, 15,393 data pairs were used for calibration and validation, and the 
corresponding scatter diagram is shown in Fig. 3(b). The colors in the two diagrams indicate the density of data pairs.

The following four statistical parameters were used to evaluate the comparisons of the ASAR-derived (referring to both raw and calibrated) sea state parameters with buoy data or RA data, where $X$ represents the ASAR-derived sea state parameters and $Y$ represents either the buoy data or the RA data. $N$ is the quantity of collocated data pairs. $\bar{X}$ and $\bar{Y}$ represent the mean values of the variables $X$ and $Y$, respectively. The correlation coefficient is calculated by the covariance $\operatorname{Cov}(X, Y)$ and the variances $D(X)$ and $D(Y)$.

$$
\begin{aligned}
\text { Correlation } & =\frac{\operatorname{Cov}(X, Y)}{\sqrt{D(X)} \sqrt{D(Y)}} \\
\operatorname{Cov}(X, Y) & =\frac{1}{N} \sum\left(X_{i}-\bar{X}\right)\left(Y_{i}-\bar{Y}\right) \\
D(X) & =\frac{1}{N} \sum\left(X_{i}-\bar{X}\right)^{2}, D(Y)=\frac{1}{N} \sum\left(Y_{i}-\bar{Y}\right)^{2} \\
\text { Bias } & =\bar{Y}-\bar{X} \\
\text { RMSE } & =\sqrt{\frac{\sum\left(Y_{i}-X_{i}\right)^{2}}{N}} \\
\text { S.I. } & =\frac{1}{Y} \sqrt{\frac{\sum\left[\left(Y_{i}-\bar{Y}\right)-(X i-\bar{X})\right]^{2}}{N}}
\end{aligned}
$$

ASAR_ $H_{s}$ is slightly higher than ECMWF_buoy_H $H_{s}$ with a bias of $0.07 \mathrm{~m}$. The RMSE is $0.62 \mathrm{~m}$, which is close to the result $(0.70 \mathrm{~m})$ achieved in the preliminary validation based on a two-month dataset ${ }^{17}$. The scatter index (S.I.) of $25.68 \%$ is relatively high. Furthermore, a comparison of the MWP results suggests that the ASAR retrievals are also slightly higher than the NDBC buoy-measured periods with a bias of $0.21 \mathrm{~s}$. In contrast, the retrieved ASAR_ $T_{m 02}$ are closely distributed both sides of the 1:1 diagonal line, and therefore, the comparison yields a low S.I. of $12.36 \%$. With respect to the correlation coefficient, both comparisons suggest that the ASAR retrievals display good agreement with the ECMWF and NDBC buoy measurements, having values of 0.89 and 0.83 , respectively.

Calibration of the ASAR-derived SWH data. Our goal is to calibrate the ASAR-derived sea state parameters using buoy measurements; however, quite a few collocations are outliers, as illustrated in Fig. 3 . If these outliers are included in the calibration process, they can introduce uncertainty. Therefore, we used quartiles to exclude some outliers from the calibration process ${ }^{29}$. Quartiles are obtained by dividing the data sorted in ascending order into four equal groups, which can be used to describe the distribution of the data and identify the outliers. The second quartile $Q_{2}$ is the median of the data. The first quartile $Q_{1}$ and the third quartile $Q_{3}$ represent the data between the median and the minimum and maximum, respectively. $I Q R$ is the interquartile range. According to $Q_{1}, Q_{2}, Q_{3}$ and the $I Q R$, the lower and upper bounds can be calculated. The data exceeding the lower and upper bounds are regarded as outliers.

$$
\begin{aligned}
I Q R & =Q_{3}-Q_{1} \\
\text { lower bound } & =Q_{1}-1.5 I Q R \\
\text { Upper bound } & =Q_{3}+1.5 I Q R
\end{aligned}
$$

By applying these quartiles to exclude some outliers, which is based on statistical analysis of the collocated data, we further employed robust regression to detect the outliers ${ }^{30,31}$ of the collocated data pairs. Robust regression is a linear regression method that is insensitive to outliers. At the start of the regression, all the fitting data have equal weights. By applying least-square minimization, the predicted values and residuals are calculated, where the residuals represent the difference between the predicted values and the observed ones. The data with large residuals are assigned small weights in the subsequent iterations. After a few iterations, the weights of the fitting data are adjusted, and the outliers are verified to have small weights. In this study, the fitting data with weights smaller 0.15 are considered outliers and are excluded from the calibration of the ASAR SWH data.

The light and dark gray cross symbols in Fig. 4 represent the outliers detected by the quantile and the robust regression methods, respectively.

Although the quantile and robust regression methods successfully excluded some data pairs as outliers (as indicated by the improved statistical parameters), the comparison shown in Fig. 4(a) suggests that the difference between ASAR_H $H_{s}$ and ECMWF_buoy_ $H_{s}$ is still distinct; specifically, the underestimation of the SWH increases along with sea state varying. In the next step, the buoy measurements were used to calibrate the ASAR retrievals.

The in situ measurements are the most appropriate data source of $\mathrm{Cal} / \mathrm{Val}$ of satellite retrievals. However, these data are not completely unbiased or free of errors ${ }^{32}$. Therefore, we used the reduced major axis (RMA) regression $\operatorname{method}^{31,33}$, which treats the variables $x\left(\right.$ ASAR_ $\left.H_{s}\right)$ and $y\left(\right.$ ECMWF_buoy_ $\left.H_{s}\right)$ independently, to calibrate the ASAR retrievals. In the regression, the errors of $x$ and $y$ are both considered by minimizing the triangular area $0.5 \times(\Delta x \Delta y)$ between the data points and the regression line, where $\Delta x$ and $\Delta y$ are the distances between the actual and predicted values in the $x$ and $y$ directions, respectively. By applying RMA regression to the collocated data pairs, the following linear calibration formula for the ASAR SWH data is obtained:

$$
\text { Calibrated_ASAR_H }(m)=1.140 \times A S A R \_H_{s}(m)-0.402
$$



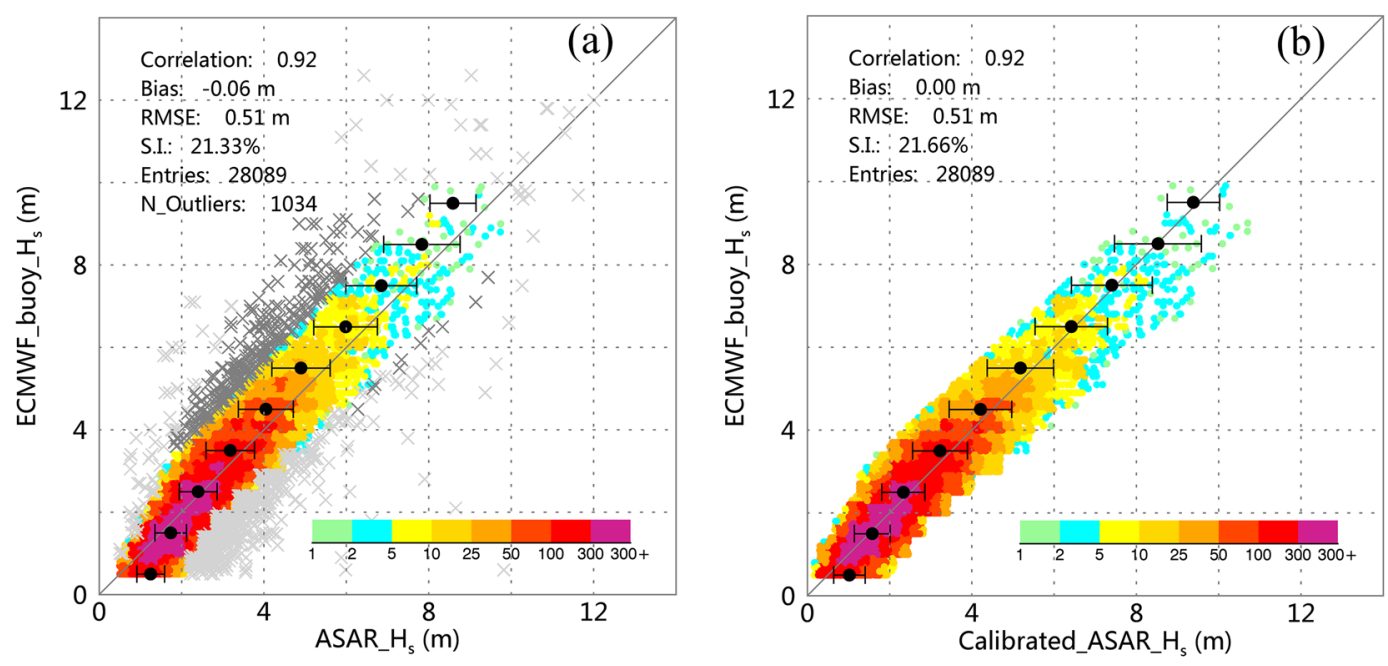

Fig. 4 (a) Comparison between the ASAR-derived SWH (excluding the detected outliers) and the ECMWF buoy SWH. The light and dark gray cross symbols are the outliers detected by quantile and robust regression methods, respectively. (b) Comparison between ASAR-derived SWH and the ECMWF buoy SWH after calibration.

\begin{tabular}{|c|c|c|c|c|c|c|}
\hline \multirow[b]{2}{*}{ Range (m) } & \multirow[b]{2}{*}{ Description } & \multirow{2}{*}{$\begin{array}{l}\text { No. } \\
\text { Collocations }\end{array}$} & \multicolumn{2}{|c|}{ Bias (m) } & \multicolumn{2}{|c|}{ RMSE (m) } \\
\hline & & & Raw & Calibrated & Raw & Calibrated \\
\hline $0.50-1.25$ & Slight & 4548 & -0.43 & -0.22 & 0.54 & 0.44 \\
\hline $1.25-2.50$ & Moderate & 13031 & -0.16 & -0.03 & 0.40 & \begin{tabular}{|l|}
0.41 \\
\end{tabular} \\
\hline $2.50-4.00$ & Rough & 7362 & 0.14 & 0.13 & 0.52 & 0.59 \\
\hline $4.00-6.00$ & Very Rough & 2622 & 0.38 & 0.18 & 0.74 & 0.75 \\
\hline $6.00-9.00$ & High & 507 & \begin{tabular}{|l|}
0.46 \\
\end{tabular} & -0.04 & \begin{tabular}{|l|}
0.89 \\
\end{tabular} & \begin{tabular}{|l|}
0.87 \\
\end{tabular} \\
\hline $9.00-14.00$ & Very High & 19 & 0.74 & -0.06 & 0.92 & 0.62 \\
\hline
\end{tabular}

Table 1. Variations in the bias (Buoy - ASAR) and RMSE with the sea state before and after the RMA calibration.

Figure 4(b) shows a comparison between the Calibrated_ASAR_ $H_{s}$ and the buoy measurements ECMWF_ buoy_ $H_{s}$. The calibration process does improve the bias, which decreases from $0.06 \mathrm{~m}$ to zero. However, the other three parameters, including the correlation coefficient, RMSE and S.I., do not improve. Although performing the calibration does not improve the overall statistical parameters, it significantly improves the underestimation of the ASAR-retrieved SWH, as revealed by the error bars overlaid on the scatter diagram, while the underestimation trend originally increases with the wave height. Because the collocated data pairs are unequally distributed among different wave heights and much of the data (62.58\%) are associated with a low to moderate sea state $(\mathrm{SWH}<2.5 \mathrm{~m})$, the overall statistical parameters do not reflect the effect of calibration on the ASAR-retrieved SWH for different sea states. The following Table 1 lists the variations in the bias and RMSE with the sea state (the Douglas sea scale is used) before and after applying the RMA calibration to the collocated data pairs.

The bias is significantly reduced by the calibration process, particularly for the slight, and higher than rough sea states. This finding indicates that the linear calibration partially reduces the problem of overestimation for slight to moderate sea states and underestimation for rough and high sea states. The RMSE displays slight fluctuations before and after the calibration process, except for the very high sea state (SWH larger than $9.00 \mathrm{~m}$ ), for which it is reduced by approximately $33 \%$ after the calibration.

Calibration of the ASAR-derived MWP. Following the same calibration method applied to the SWH, the NDBC buoy data are used to calibrate the ASAR-derived MWP. In total, 15,393 data pairs were collected to calibrate the MWP data considering the collocation criteria mentioned above. After elimination of outliers by the quartile and robust regression methods, 14,970 pairs of data remained. The scatter diagram of the comparison is shown in Fig. 5(a), where the colors represent the density of data pairs and the cross symbols indicate the detected outliers. Using the RMA regression method, a linear calibration of the MWP is derived:

$$
\text { Calibrated_ASAR_T } T_{m 02}(s)=1.268 \times A S A R \_T_{m 02}(s)-1.887
$$

The calibrated ASAR MWP results are plotted against the NDBC buoy data in Fig. 5(b). Comparing Fig. 5(a) with (b), the calibration improves both the bias and the RMSE, which decrease from $-0.19 \mathrm{~s}$ to zero and from $0.67 \mathrm{~s}$ to $0.65 \mathrm{~s}$, respectively. However, the correlation coefficient and S.I. do not improve. The raw data suggest that 

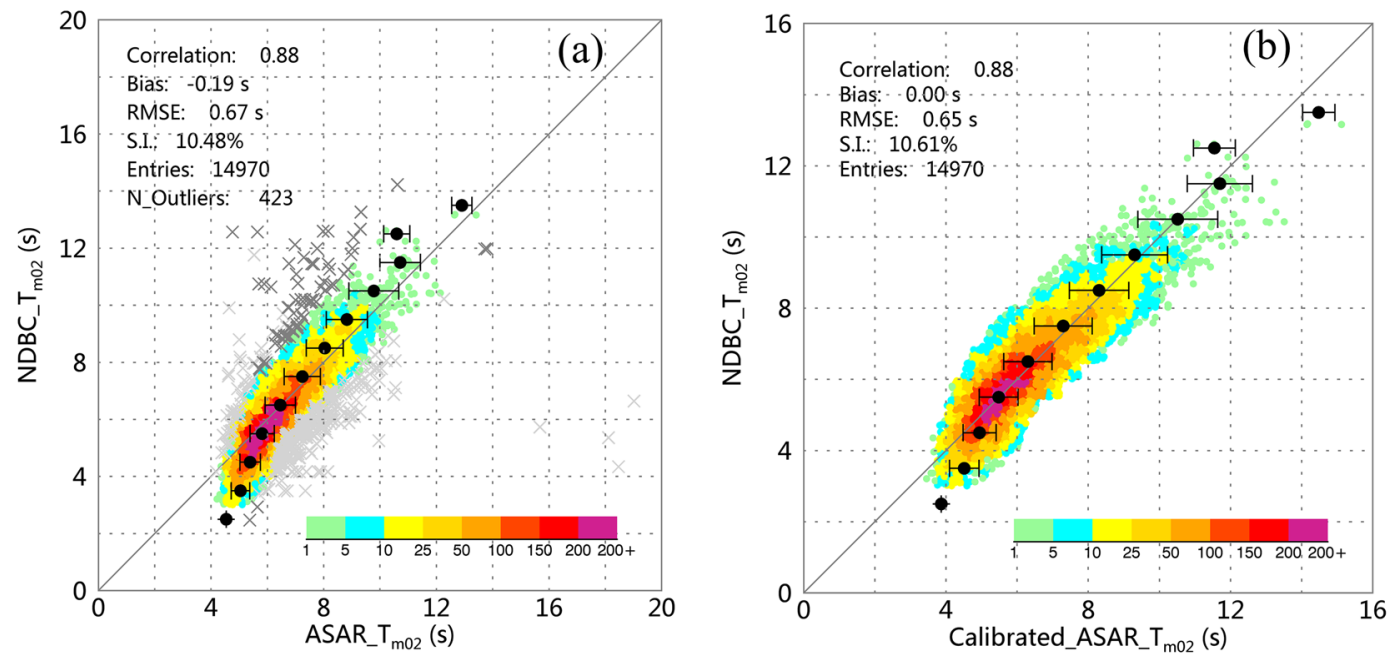

Fig. 5 (a) Scatter diagram of the comparison between the ASAR-derived MWP and the NDBC buoy MWP. (b) the same as (a) but for the comparison of the calibrated ASAR-derived MWP. There are few data pairs with large values of MWP that are detected outliers (lower right of (a)); therefore, the maximum value of the axes in (b) is reduced to $16 \mathrm{~s}$ to better visualize the distribution of the error bars. The cross symbols in (a) are the detected outliers using the IQR (light gray) and robust regress (dark gray) methods.

the ASAR-derived results overestimate the MWP below $7 \mathrm{~s}$ but underestimate it above $8 \mathrm{~s}$. The calibration makes the data pairs almost symmetrically distributed about the 1:1 diagonal line and partially corrects the trend result.

The above-described calibration of the ASAR retrieval is based on collocating buoy data within a $100 \mathrm{~km}$ distance. We also tried to reduce the collocation distance to $50 \mathrm{~km}$ (consequently, the number of collocation data pairs decreased to 8,046), which yields the following two calibration formulas for the SWH and MWP, respectively, using the same method described above.

$$
\begin{aligned}
& \text { Calibrated_ASAR_H} H_{s}(m)=1.132 \times A S A R \_H_{s}(m)-0.383 \\
& \text { Calibrated_ASAR_T } T_{m 02}(s)=1.256 \times A S A R \_T_{m 02}(s)-1.821
\end{aligned}
$$

The difference in the formulas derived based on a $100 \mathrm{~km}$ and $50 \mathrm{~km}$ collocation distance for calibrating the ASAR-derived SWH and MWP is nearly neglected. For instance, assuming an extreme sea state with the SWH of $20 \mathrm{~m}$, the difference in the calibrated SWH using the two formulas is approximately $0.14 \mathrm{~m}$, which accounts for $0.7 \%$ of the SWH. In the provided product, the derived calibration formulas based on a $100 \mathrm{~km}$ collocation distance are applied to the ASAR retrieval of SWH and MWP, while the user can easily apply the other set of calibration formulas in (9) and (10) for exploitation.

\section{Data Records}

The ASAR WM data global wave product is stored in NetCDF-3 format and follows the Climate and Forecast Metadata CF-1.7 convention ${ }^{34}$. The naming convention of the ASAR sea state product files is as follows:

Satid_Sensor_Type_StartDate_StartTime_EndDate_EndTime_Cycle_Orbit.NC, where

a. Satid: mission name

b. Sensor: sensor name

c. Type: type of product

d. StartDate: Date of the first record

e. StartTime: Time of the first record

f. EndDate: Date of the last record

g. EndTime: Time of the last record

h. Cycle: cycle number of the satellite

i. Orbit: relative orbit number of the satellite

The records contained in the product correspond to the imagettes of the ASAR WVI Level 1B product. Each record consists of 14 variables, which are listed in the following Table 2.

The "swh" and " $m w p$ " are the retrieved sea state parameters using the CWAVE_ENV model. By applying the calibration formulas given in Eqs. (7) and (8), the calibrated ASAR-derived SWH and MWP are obtained and stored as the variables "swh_cali" and " $m w p \_c a l i "$.

The diagram shown in Fig. 6 illustrates the structure of the "rejection_flag" and "qc_flag" designed in the product.

The "rejection_Flag" flags mark the ASAR WM records with values of $0,1,2,3,4,5$ or 6 , which represent an acceptable ASAR WM imagette for retrieval, a bad record (identified in reading the Level 1B data), a record 


\begin{tabular}{|c|c|c|}
\hline No. & Variables & Description \\
\hline 1 & time & $\begin{array}{l}\text { Acquisition time of the ASAR imagettes. Seconds since } \\
\text { 2000-01-01 00:00:00 UTC }\end{array}$ \\
\hline 2 & latitude & Latitude of ASAR imagette center \\
\hline 3 & longitude & Longitude of ASAR imagette center \\
\hline 4 & heading & Flight direction of the satellite (clockwise relative to north) \\
\hline 5 & inci_angle & Local incidence angle of ASAR imagette center \\
\hline 6 & land_flag & $\begin{array}{l}0 \mathrm{~B} \text { for ocean area } \\
1 \mathrm{~B} \text { for land area }\end{array}$ \\
\hline 7 & homogeneity & Homogeneity of ASAR imagettes \\
\hline 8 & normalized_variance & Normalized variance of ASAR imagettes \\
\hline 9 & rejection_flag & $\begin{array}{l}\text { The records flagged } 0 \mathrm{~B} \text { are acceptable ASAR imagettes for } \\
\text { retrieval } \\
\text { 1B for a "bad_record" } \\
\text { 2B for "land" } \\
\text { 3B for "inhomogeneous ASAR imagettes" } \\
\text { 4B for "ASAR imagettes in HH polarization" } \\
\text { 5B for "ASAR imagettes with an incidence angle not equal } \\
\text { to } 23^{\circ} \text { " } \\
6 \mathrm{~B} \text { for "ASAR imagettes in polar regions, i.e. beyond } 70^{\circ} \mathrm{N} \\
\text { or } 65^{\circ} \mathrm{S} \text { " }\end{array}$ \\
\hline 10 & qc_flag & $\begin{array}{l}0 \mathrm{~B} \text { for a good record } \\
1 \mathrm{~B} \text { for a suspect record } \\
2 \mathrm{~B} \text { for a bad record }\end{array}$ \\
\hline 11 & swh & Retrieved SWH of ASAR imagettes \\
\hline 12 & mwp & Retrieved zero-upcrossing wave period of ASAR imagettes \\
\hline 13 & swh_cali & Calibrated SWH \\
\hline 14 & mwp_cali & Calibrated zero-upcrossing MWP \\
\hline
\end{tabular}

Table 2. List of variables and their descriptions in the ASAR WM sea state NetCDF product.

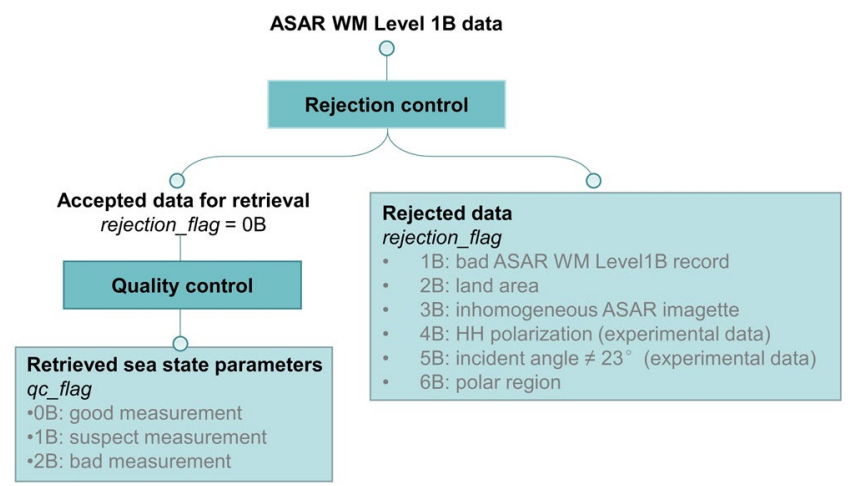

Fig. 6 Structure of the "rejection_flag" and " $q$ c_flag" of the developed global ASAR WM wave products.

containing land (discrimination is based on "land_flag"), an inhomogeneous (homogeneity factor $>1.5$ ) ASAR imagette, an imagette acquired in $\mathrm{HH}$ polarization, an imagette with an incidence angle not equal to $23^{\circ}$, and an imagette acquired in the polar regions, respectively. The "land_flag" is inherited from the ASAR WM Level 1B data, i.e., each imagette in the Level $1 \mathrm{~B}$ data has been flagged "land" or not. The "normalized_variance" 25 variable is the normalized variance of the ASAR WM intensity data and is calculated according to Eq. (11).

$$
\begin{aligned}
\text { noramalized_variance } & =\frac{I_{\text {var }}}{I_{\text {mean }} \times I_{\text {mean }}} \\
I_{\text {mean }} & =\frac{\sum_{i, j=1}^{M, N} I_{i, j}}{M \times N} \\
I_{\text {var }} & =\frac{\sum_{i, j=1}^{M, N}\left(I_{i, j}-I_{\text {mean }}\right)^{2}}{M \times N}
\end{aligned}
$$

where $I_{v a r}$ and $I_{\text {mean }}$ represent the variance and mean of the image, respectively, and $M$ and $N$ refer to the width and height of the image, respectively.

The " $q$ c_flag" variable has three values that describe the quality of the retrieved sea state parameters. We considered a few factors during the quality control process, including the reasonable range of retrievals, the 
normalized variance of the original ASAR intensity image and the "rejection_flag". Based on the factors, the records were assigned different flags.

(1) Good record $\left(q c \_f l a g=0 \mathrm{~B}\right)$, which satisfies the following criteria:

a. $0 \mathrm{~m} \leq s w h\left(s w h \_c a l i\right)<30 \mathrm{~m}$ and $0 \mathrm{~s}<m w p\left(m w p \_c a l i\right)<20 \mathrm{~s}$

b. $\overline{\sigma_{0}}-\mathrm{NESZ}>3 \mathrm{~dB}$

c. rejection_flag $=0 \mathrm{~B}$

d. homogeneity factor $<1.05$

where $\bar{\sigma}_{0}$ is the mean normalized radar cross section of the ASAR imagettes and NESZ is the noise equivalent sigma zero, i.e., the noise floor of the ASAR WM data.

(2) Suspect record $\left(q c \_\right.$flag $\left.=1 \mathrm{~B}\right)$, which satisfies the following criterion:

a. $s w h>30 \mathrm{~m}$ or $m w p>20 \mathrm{~s}$

b. $1.05 \leq$ homogeneity factor $\leq 1.5$

Among the ASAR collocations with buoy data, there are 1,416 data pairs with homogeneity factors between 1.05 and 1.50. Their comparison with the ECMWF buoy SWH has a bias of $-0.26 \mathrm{~m}$, an RMSE of $1.02 \mathrm{~m}$, and a correlation of 0.63 . Although these statistical parameters are obviously worse than the comparison achieved using the ASAR WM data with homogeneity factors less than 1.05 (Fig. 3(a)), a large portion of these data still have good consistency with the buoy measurements. If the collocation data pairs with homogeneity factors larger than 1.50 (the amount is 677) are compared with the ECMWF buoy SWH, a correlation of only 0.31 and a large S.I. of $79.20 \%$ are found. Therefore, we assign the " $q$ c_flag" of the ASAR retrievals with homogeneity factors between 1.05 and 1.50 to "suspect", indicating that these records require further investigation.

(3) Bad record ( $q$ c_flag $=2 \mathrm{~B})$, which satisfies one of the following conditions:

a. $s w h\left(s w h \_c a l i\right)<0 \mathrm{~m}$ or $m w p\left(m w p \_c a l i\right)<0 \mathrm{~s}$

b. $\bar{\sigma}_{0}-\mathrm{NESZ} \leq 3 \mathrm{~dB}$

Any record with the variable "rejection_flag" not equal to $0 \mathrm{~B}$ is excluded from further processing, and the "qc_flag" is therefore set to "_Fillvalue".

Each ASAR WM Level $1 \bar{B}$ data with a filename extension of "N1" that we received from the ESA is processed to a NC record. All the NC records in the same year are compressed to a single file with the file extension ".tar.gz"; therefore, there are together 11 GNU zip files corresponding to the data from the year 2002 to 2012 . They have been uploaded to the public repository Sea Scientific open data publication (SEANOE, https://www.seanoe.org) with full free access ${ }^{35}$.

\section{Technical Validation}

Comparison with RA wave data. The GlobWave project also collected wind and wave data for the Geodetic Satellite (GEOSAT), GEOSAT Follow-on (GFO), ERS-1, ERS-2, TOPEX/POSEIDON, JASON-1, JASON-2 and CryoSAT-2 RA missions, with a time span from 1985 onwards. The JASON-1 mission provided wave data from December 2001 until July 2013, which covers the lifetime of the ASAR instrument. GlobWave reprocessed the original JASON-1 measurements and provided quality control flags and calibrated SWH measurements. In this study, we used calibrated Ku-band SWH measurements of JASON-1 to perform a cross-validation with the calibrated ASAR-derived SWH. A quality flag named "swh_quality" provided in the GlobWave RA products is used to filter the JASON-1 SWH data with high quality for validation. This flag has three values, namely, 0,1 , and 2, representing a "good_measurement", "acceptable_for_some_applications" and "bad_measurement", respectively. Only the data flagged as "good_measurement" are used for validation. The same collocation criteria employed in the collocation of buoy data were utilized between the ASAR data and the JASON-1 data.

Following the same criteria of collocating the ASAR with buoy, 46,642 data pairs of JASON-1 and ASAR WM data were obtained. However, a large number of SWH measurements from JASON-1 of the GlobWave product in 2012 were abnormal, ranging from $-40 \mathrm{~m}$ to $40 \mathrm{~m}$ and exhibiting a discontinuous spatial distribution. The data in 2012 were discarded from the validation dataset. In addition, we set a valid range of SWH from $0.5 \mathrm{~m}$ to $30 \mathrm{~m}$ for validation. Finally, 23,192 pairs of JASON-1 and ASAR WM data were obtained. Using the quartile method described above to exclude outliers, 22,880 pairs of data were collected for validation. As there are only few data available for SWH above $10 \mathrm{~m}$, the quartile method of detecting outliers does not function in that range. However, we retain them for comparison. Figure 7(a) shows the comparison between the ASAR-derived SWH and JASON-1 SWH (denoted JASONI_Hs). The robust regression method was not applied to exclude outliers because we consider both datasets to comprise independent measurements. The calibrated ASAR SWH (applying Eq. (7)) is also compared with the JASON-1 calibrated SWH ${ }^{35}$ (Calibrated_JASONI_Hs), as shown in Fig. 7(b).

As shown in Fig. 7(a), the ASAR SWH displays good consistency with the JASON-1 SWH, and the bias and RMSE are $0.04 \mathrm{~m}$ and $0.48 \mathrm{~m}$, respectively; additionally, the correlation coefficient and S.I. are 0.93 and $16.84 \%$, respectively. Although the ASAR SWH is generally slightly lower than the JASON-1 SWH, it is higher for a relatively low sea state (SWH $<2.5 \mathrm{~m}$ ). In Fig. 7(b), the calibrated ASAR SWH also displays good agreement with the calibrated JASON-1 SWH, with bias, RMSE, correlation coefficient and S.I. values of $0.18 \mathrm{~m}, 0.53 \mathrm{~m}, 0.93$ and 

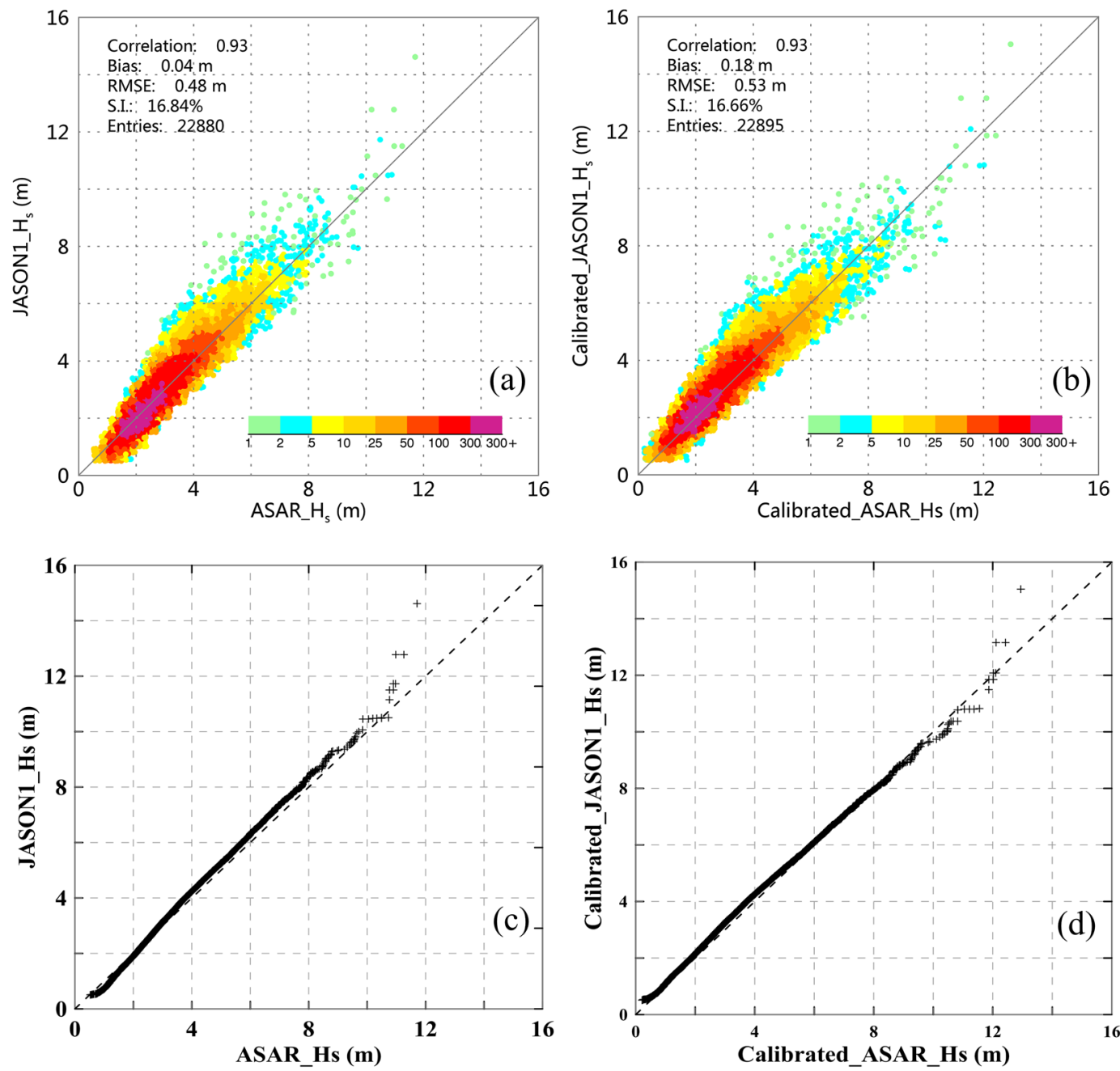

Fig. 7 (a,c) Comparison between the ASAR-derived SWH and the RA SWH along with the corresponding Q-Q plot, respectively. (b,d) Comparison between the calibrated ASAR-derived SWH and the calibrated RA SWH along with the corresponding Q-Q plot, respectively.
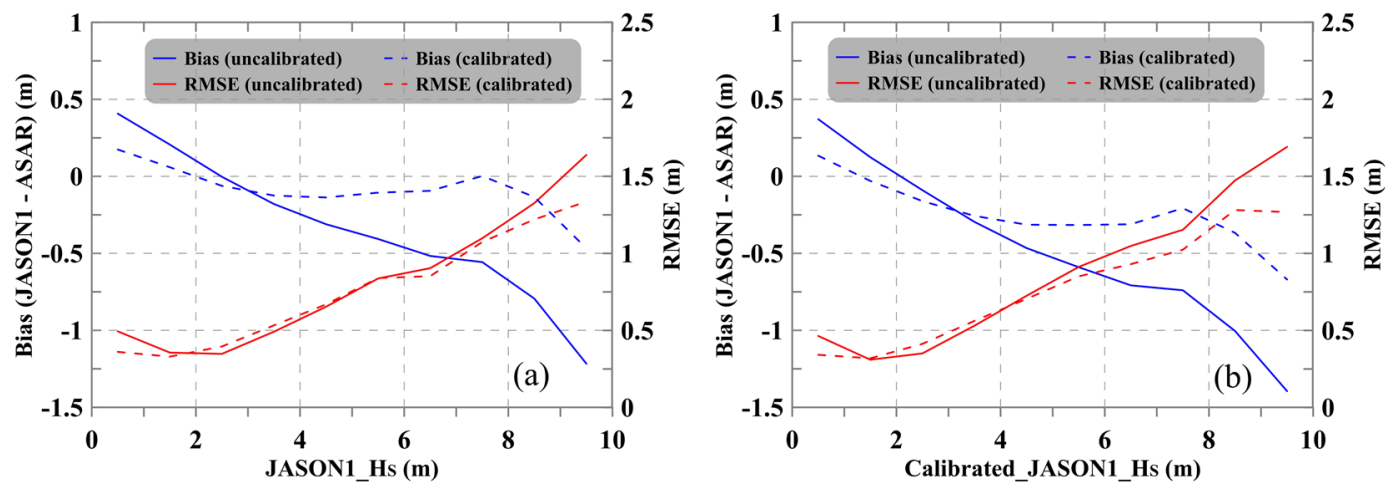

Fig. 8 Variations in the bias and RMSE of the ASAR-derived SWH versus the JASON-1 SWH (a) and the calibrated JASON-1 SWH (b).

16.64\%, respectively. The Q-Q (quantile-quantile) plots shown in Fig. 7(c,d) suggest that the underestimation of ASAR-derived SWH is significantly improved after the calibration process, particularly for SWH above $6 \mathrm{~m}$.

A major limitation of these overall comparisons in evaluating the retrieval of sea state parameters is that the data pairs are unevenly distributed among different sea states. As the sea state increases in severity, the number of valid data pairs decreases. Therefore, a stepwise comparison was conducted to assess the performance of the 
ASAR SWH data quality for different sea states. Figure 8(a) shows the uncalibrated and calibrated ASAR SWH compared with the JASON-1 SWH at a 1-m interval. Figure 8(b) is the same as (a) but compares the ASAR SWH with the calibrated JASON-1 SWH.

Due to the changes in the bias and RMSE illuminated in Fig. 8(a,b) showing similar trends, we use Fig. 8(a) as an example for further description. Figure 8(a) shows the changes in the bias and RMSE of the uncalibrated and calibrated ASAR SWH versus the JASON-1 SWH, where the blue and red lines represent the bias and RMSE, respectively and the solid and dashed lines refer to the comparisons based on the uncalibrated and calibrated ASAR SWH, respectively. The bias of the uncalibrated ASAR SWH increases with the sea state and changes from negative to positive when the SWH is approximately $2 \mathrm{~m}$. The calibration process significantly reduces the bias to less than $0.15-0.2 \mathrm{~m}$ from low to high sea states (at approximately $8 \mathrm{~m}$ ), and importantly, the bias becomes less dependent on the sea state increasing. For a very high sea state (SWH $>9 \mathrm{~m}$ ), the bias accounts for approximately $10 \%$ of the total SWH; additionally, the RMSE of the calibrated ASAR SWH varies from $0.25 \mathrm{~m}$ to $1.20 \mathrm{~m}$ and is particularly reduced for sea states higher than very rough (above approximately $5 \mathrm{~m}$ ).

The cross-validation of the ASAR-derived SWH is based on the comparison with the GlobWave JASON-1 data mainly due to that the JASON-1 RA wave data has almost the same temporal coverage as the ASAR WM data in full-life time. Cross-validations with other RA data remain further investigation, e.g., using the recently released comprehensive RA dataset by Ribal and Young ${ }^{1}$, in which there are a few RA missions that also have overlap with the operating period of the ASAR. This can particularly diagnose the accuracy of the ASAR SWH of high sea state, as found in Fig. 7.

Thus far, there is no other high-quality MWP dataset by spaceborne remote sensing available for cross-validation of the ASAR-derived MWP. Further investigation by carefully selecting a reanalysis wave model dataset might be worth trying.

\section{Code availability}

Both the MATLAB code script named read_AGWD.m and the IDL code named read_AGWD.pro for reading the ocean wave parameter products are provided as supplementary material 1 and 2, respectively.

Received: 22 January 2020; Accepted: 14 July 2020;

Published online: 07 August 2020

\section{References}

1. Ribal, A. \& Young, I. R. 33 years of globally calibrated wave height and wind speed data based on altimeter observations. Scientific Data. 6, 1-15 (2019).

2. Young, I. R., Zieger, S. \& Babanin, A. V. Global trends in wind speed and wave height. Science. 332, 451-455 (2011).

3. Brown, W. M. \& Procello, C. J. An introduction to synthetic aperture radar. IEEE spectrum. 6, 57-62 (1969).

4. Born, G. H., Dunne, J. A. \& Lame, D. B. Seasat mission overview. Science. 204, 1405-1406 (1979).

5. McLeish, W. et al. Synthetic Aperture Radar imaging of ocean waves: Comparison with wave measurements. J. Geophys. Res. 85, 5003-5011 (1980).

6. Alpers, W. \& Rufenach, C. L. The effect of orbital motions of Synthetic Aperture Radar imagery of ocean waves. IEEE transactions on Antennas and Propagation. 27, 685-690 (1979).

7. Hasselmann, K. et al. Theory of Synthetic Aperture Radar ocean imaging: A MARSEN view. J. Geophys. Res. 90, 4659-4686 (1985).

8. Hasselmann, K. \& Hasselmann, S. On the nonlinear mapping of an ocean wave spectrum into a synthetic aperture radar image spectrum. J. Geophys. Res. 96, 10713-10729 (1991).

9. WAMDI GROUP. The WAM model a third generation ocean wave prediction model. J. Phys. Oceanogry. 18, 1775-1810 (1984).

10. Mastenbroek, C. \& de Valk, C. A semi-parametric algorithm to retrieve ocean wave spectra from synthetic aperture radar. J. Geophys. Res. 105, 3497-3516 (1998).

11. Hasselmann, S., Brüning, C., Hasselmann, K. \& Heimbach, P. An improved algorithm for the retrieval of ocean wave spectra from synthetic aperture radar image spectra. J. Geophys. Res. 101, 16615-16629 (1996).

12. Kerbaol, V., Chapron, B. \& Vachon, P. W. Analysis of ERS-1/2 synthetic aperture radar wave mode imagettes. J. Geophys. Res. 103, 7833-7846 (1998).

13. Lehner, S., Schulz-Stellenfleth, J., Schättler, J. B., Breit, H. \& Horstmann, J. Wind and wave measurements using complex ERS-2 wave mode data. IEEE Trans. Geosci., and Rem. Sens. 38, 2246-2257 (2000).

14. Schulz-Stellenfleth, J., König, Th. \& Lehner, S. An empirical approach for the retrieval of integral ocean wave parameters from synthetic aperture radar data. J. Geophys. Res. 112, (2007).

15. Hasselmann, K. et al. The ERS SAR wave mode: a breakthrough in global ocean wave observations. ESA special publication. SP-1326, 167-197 (2012)

16. Li, X.-M., König, T., Schulz-Stellenfleth, J. \& Lehner, S. Validation and intercomparison of ocean wave spectra retrieval scheme using ASAR wave mode data. International Journal of Remote Sensing. 31, 4969-4993 (2010).

17. Li, X.-M., Lehner, S. \& Bruns, T. Ocean wave integral parameter measurements using Envisat ASAR wave mode Data. IEEE Trans. Geosci. Remote Sens. 49, 155-174 (2011).

18. Stopa, J. E. \& Mouche, A. Significant wave heights from Sentinel-1 SAR: Validation and applications. J. Geophys. Res. 122, 1827-1848 (2017).

19. Li, X.-M., Zhang, T. Y., Huang, B. Q. \& Jia, T. Capabilities of Chinese Gaofen-3 synthetic aperture radar in selected topics for coastal and ocean observations. Remote Sens. 10, 1-22 (2018).

20. Ardhuin, F., Chapron, B. \& Collard, F. Observation of swell dissipation across oceans. Geophys. Res. Lett. 36, L06607 (2009).

21. Collard, F., Ardhuin, F. \& Chapron, B. Monitoring and analysis of ocean swell fields from space: New methods for routine observations. J. Geophys. Res. 114, C07023 (2009).

22. Li, X.-M. A new insight from space into swell propagation and crossing in the global oceans. Geophys. Res. Lett. 43, 5202-5209 (2016).

23. Chapron, B., Johnson, H. \& Garello, R. Wave and wind retrieval from SAR images of the ocean. Ann. Telecommun. 56, 682-699 (2001).

24. Engen, G. \& Johnson, H. SAR-ocean wave inversion using image cross spectra. IEEE Trans. Geosci. Remote Sens. 33, 329-360 (2000).

25. Brooker, G. UWA processing algorithm specification. version 2.0, Tech. Rep., ESA, ESTEC/NWP, Noordwijk, The Netherland, 1995.

26. European Space Agency, ENVISAT ASAR Product Handbook, Issue 2.2, 2007. 
27. Stoffelen, A. \& Anderson, D. Scatterometer data interpretation: Estimation and validation of the transfer function CMOD4. J. Geophys. Res. 102, 5767-5780 (1997).

28. Schulz-Stellenfleth, J. \& Lehner, S. Measurement of 2-D sea surface elevation fields using complex Synthetic Aperture Radar data. IEEE Trans. Geosci., \& Rem. Sens. 42, 1149-1160 (2004).

29. Tukey, J. Exploratory Data Analysis. (Addison-Wesley. 1977)

30. Rousseeuw, P. \& Leroy, A. Robust Regression and Outlier Detection 3rd edn (John Wiley \& Sons, 1996)

31. Zieger, S., Vinoth, J. \& Young, I. R. Joint calibration of multiplatform altimeter measurements of wind speed and wave height over the past 20 years. Journal of Atmospheric and Oceanic Technology. 26, 2549-2564 (2009).

32. Stoffelen, A. Toward the true near-surface wind speed: Error modeling and calibration using triple collocation. J. Geophys. Res. 103, 7755-7766 (1998).

33. Trauth, M. MATLAB Recipes for Earth Sciences. 4th Edn (Springer, 2015).

34. Eaton, B., Gregory, J. \& Bod Drach etc. NetCDF Climate and Forecast (CF) Metadata Convebtions Version 1.7.

35. Li, X.-M. \& Huang, B. Q. A global sea state dataset from spaceborne synthetic aperture radar wave mode data. SEANOE https://doi. org/10.17882/71337 (2020).

\section{Acknowledgements}

The first author would like to thank Dr. Susanne Lehner and Dr. Johannes Schulz-Stellenfleth, my supervisor and former colleague for their supports to develop the CWAVE_ENV parametric model for the ASAR wave mode data. We particularly thank the ESA for agreeing to deliver us the entire ASAR wave mode dataset under the framework of the "Dragon 4 program". Mr. Jean-Francois Piollo from Ifremer/Cersat gave us the cordial help of delivering these wave mode data. Dr. Alexis Mouche, also from Ifremer/Cersat, encouraged us to participate the ESA sea state CCI project. This is one of our motivations to reprocess the entire ASAR WM dataset for times to standard ocean wave products. The study is partially supported by the National Key Research and Development Project (2018YFC1407100) and the National Natural Science Foundation of China (41406198).

\section{Author contributions}

X.L. initiated, designed and implemented the project and performed the bulk of the work creating the dataset, conducting the evaluations and writing the paper. B.H. worked on the software to produce the products and performed the calibration and validation tasks. She also contributed to drafting the manuscript.

\section{Competing interests}

The authors declare no competing interests.

\section{Additional information}

Supplementary information is available for this paper at https://doi.org/10.1038/s41597-020-00601-3.

Correspondence and requests for materials should be addressed to X.-M.L.

Reprints and permissions information is available at www.nature.com/reprints.

Publisher's note Springer Nature remains neutral with regard to jurisdictional claims in published maps and institutional affiliations.

(c) (i) Open Access This article is licensed under a Creative Commons Attribution 4.0 International License, which permits use, sharing, adaptation, distribution and reproduction in any medium or format, as long as you give appropriate credit to the original author(s) and the source, provide a link to the Creative Commons license, and indicate if changes were made. The images or other third party material in this article are included in the article's Creative Commons license, unless indicated otherwise in a credit line to the material. If material is not included in the article's Creative Commons license and your intended use is not permitted by statutory regulation or exceeds the permitted use, you will need to obtain permission directly from the copyright holder. To view a copy of this license, visit http://creativecommons.org/licenses/by/4.0/.

The Creative Commons Public Domain Dedication waiver http://creativecommons.org/publicdomain/zero/1.0/ applies to the metadata files associated with this article.

(C) The Author(s) 2020 